\title{
Adaptive Importance Sampling in General Mixture Classes*
}

\author{
Olivier Cappé, \\ LTCI, TELECOM ParisTech, CNRS \\ RANDAL Douc, \\ TELECOM SUdParis \\ Arnaud Guillin, \\ LATP, Ecole Centrale Marseille, CNRS \\ JeAn-Michel Marin, \\ Project SELECT, INRIA Saclay, Université Paris Sud \& CREST, INSEE \\ \& Christian P. ROBert \\ CEREMADE, Université Paris Dauphine, CNRS \& CREST, INSEE
}

\begin{abstract}
In this paper, we propose an adaptive algorithm that iteratively updates both the weights and component parameters of a mixture importance sampling density so as to optimise the performance of importance sampling, as measured by an entropy criterion. The method, called M-PMC, is shown to be applicable to a wide class of importance sampling densities, which includes in particular mixtures of multivariate Student $t$ distributions. The performance of the proposed scheme is studied on both artificial and real examples, highlighting in particular the benefit of a novel Rao-Blackwellisation device which can be easily incorporated in the updating scheme.

Keywords: Importance sampling, Adaptive Monte Carlo, Mixture model, Entropy, KullbackLeibler divergence, EM algorithm, Population Monte Carlo.
\end{abstract}

\section{Introduction}

In recent years, there has been a renewed interest in using Monte Carlo procedures based on Importance Sampling (abbreviated to IS in the following) for inference tasks. Compared to alternatives such as Markov Chain Monte Carlo methods, the main appeal of IS procedures lies in the possibility of developing parallel implementations, which becomes more and more important with the generalisation of multiple core machines and computer clusters. Importance sampling procedures are also attractive in that they allow for an easy assessment of the Monte Carlo error (provided trustworthy estimates of the variance can be produced). As a consequence, it is therefore easier to construct learning mechanisms in IS settings because of this ability to compare the errors. In many applications, the fact that IS procedures may be tuned - by choosing an appropriate IS density - to minimise the approximation error for a specific function of interest is also crucial. On the other hand, the shortcomings of IS approaches are also well-known, including a poor scaling to highly multidimensional problems and an acute sensitivity to the choice of the IS density combined with the fact that it is impossible to come up with a universally efficient IS density. While there exist a wide variety of

${ }^{*}$ This work has been supported by the Agence Nationale de la Recherche (ANR) through the 2006-2008 project Adap'MC. Both last authors are grateful to the participants to the BIRS 07w5079 meeting on "Bioinformatics, Genetics and Stochastic Computation: Bridging the Gap", Banff, for their comments on an earlier version of this paper. The last author also acknowledges an helpful discussion with Geoff McLachlan. The authors wish to thank both referees for their encouraging comments. 
solutions in the literature (see, e.g. Robert and Casella, 2004, Chapter 14), this paper concentrates on the construction of adaptive importance sampling schemes in which the IS density is gradually improved based on the outcome of previous Monte Carlo draws.

While the method proposed here can be traced back to authors such as West (1992) or Oh and Berger (1993), it is closely related to the so-called Population Monte Carlo (henceforth abbreviated to PMC) approach - in the sense of an iterated simulation of importance samples and in opposition to Markov Chain Monte Carlo simulation that only produces a point at a time -introduced by Cappé et al. (2004). We briefly review the PMC approach, following the exposition of Cappé et al. (2004) and Douc et al. (2007a, b) , in order to highlight the differences with the present work. In PMC, a sample $\left(X_{1}, \ldots, X_{N}\right)$ approximately distributed from $\pi$, is repeatedly perturbed stochastically using an arbitrary Markov transition kernel $q\left(x, x^{\prime}\right)$ so as to produce a new sample $\left(X_{1}^{\prime}, \ldots, X_{N}^{\prime}\right)$. Conducting a resampling step based on the IS weights $\omega_{i}=\pi\left(X_{i}^{\prime}\right) / q\left(X_{i}, X_{i}^{\prime}\right)$, it is then possible to produce a new unweighted sample $\left(\tilde{X}_{1}, \ldots, \tilde{X}_{N}\right)$ that also constitutes an approximation to the target distribution $\pi$. Adaptivity in PMC was achieved by considering a transition kernel $q$ consisting of a mixture of fixed transition kernels

$$
q_{\alpha}\left(x, x^{\prime}\right)=\sum_{d=1}^{D} \alpha_{d} q_{d}\left(x, x^{\prime}\right), \quad \sum_{d=1}^{D} \alpha_{d}=1,
$$

whose weights $\alpha_{1}, \ldots, \alpha_{D}$ are tuned adaptively, along the iteration of the PMC algorithm. The adaptation procedure proposed by Douc et al. (2007a)), termed D-kernel PMC, aims at minimising the deviance or entropy criterion between the kernel $q_{\alpha}$ and the target $\pi$,

$$
\mathfrak{E}\left(\pi, q_{\alpha}\right)=\mathbb{E}_{\pi}^{X}\left[D\left(\pi \| q_{\alpha}(X, \cdot)\right)\right],
$$

where $D(p \| q)=\int \log \{p(x) / q(x)\} p(x) \mathrm{d} x$ denotes the Kullback-Leibler divergence (also called relative entropy), and where the expectation is taken under the target distribution $X \sim \pi$ since the kernels $q_{d}\left(x, x^{\prime}\right)$ depend on the starting value $x$. In the sequel, we refer to the criterion in (2) as the entropy criterion since it is obviously related to the performance measure used in the cross-entropy method of Rubinstein and Kroese (2004). In Douc et al. (2007b), a version of this algorithm was developed to minimise the asymptotic variance of the IS procedure, for a specific function of interest, in lieu of the entropy criterion.

A major limitation in the approaches of Douc et al. $(2007 \mathrm{a}, \mathrm{b})$ is that the proposal kernels $q_{d}$ remain fixed over the iterative process while only the mixture weights $\alpha_{d}$ get improved. In the present contribution, we remove this limitation by extending the framework of Douc et al. (2007a) to allow for the adaption of IS densities of the form

$$
q_{(\alpha, \theta)}(x)=\sum_{d=1}^{D} \alpha_{d} q_{d}\left(x ; \theta_{d}\right),
$$

with respect to both the weights $\alpha_{d}$ and the internal parameters $\theta_{d}$ of the component densities. The proposed updating mechanism is quite similar to the EM algorithm with the E-step replaced by IS computations. As demonstrated through the example considered in Section 4 , this adaptive scheme is applicable to very general families of latent-data IS densities. A possible drawback of adapting the internal parameters $\theta_{d}$ of the component densities is that it sometimes raises challenging robustness issues, particularly when (multidimensional) scaling parameters are tuned. We thus propose a Rao-Blackwellisation scheme that empirically appears to be very efficient while inducing a modest additional algorithmic complexity.

Note again that we consider here the generic entropy criterion of Douc et al. (2007a) rather than the function-specific variance minimisation objective of Douc et al. (2007b). This choice is motivated by the recognition that in most applications, the IS density is expected to perform well 
for a range of typical functions of interest rather than for a specific target function $h$. In addition, the generalisation of the approach of Douc et al. (2007b) to a class of mixture IS densities that are parameterised by more than the weights remains an open question (see also Section 5). A second remark is that in contrast to the previously cited works and as obvious in equation (3), we consider in this paper only "global" independent IS densities. Thus, the proposed scheme is based on genuine iterated importance sampling, contrary to what happens when using more general IS transition kernels as in (11). Obviously, resorting to moves that depend on the current sample is initially attractive because it allows for some local moves as opposed to the global exploration required by independent IS densities. However, the fact that the entropy criterion in (2) is a global measure of fit tends to modify the parameters of each transition kernel depending on its average performance over the whole sample, rather than locally. In addition, structurally imposing a dependence on the points sampled at the previous iteration induces some extra-variability which can be detrimental when more parameters are to be estimated.

The paper is organised as follows: In Section 2, we develop a generic updating scheme for independent IS mixtures (3), establishing that the integrated EM argument of Douc et al. (2007a) remains valid in our setting. Note once again that the integrated EM update mechanism we uncover in this paper is applicable to all missing data representations of the proposal kernel, and not only to finite mixtures. In Section 3, we consider the case of Gaussian mixtures which naturally extend the case of mixtures of Gaussian random walks with fixed covariance structure considered in Douc et al. $(2007 \mathrm{a}, \mathrm{b})$. In Section 4 , we show that the algorithm also applies to mixtures of multivariate $t$ distributions with the continuous scale mixing representation used in Peel and McLachlan (2000). Section 5 provides some conclusive remarks about the performance of this approach as well as possible extensions.

\section{Adapting the Importance Sampling Density}

\subsection{The M-PMC Algorithm}

When considering independent mixture IS densities of the form (3), the entropy criterion $\mathfrak{E}$ defined in (2) reduces to the Kullback-Leibler divergence between the target density $\pi$ and the mixture $q_{(\alpha, \theta)}$ :

$$
\mathfrak{E}\left(\pi, q_{(\alpha, \theta)}\right)=D\left(\pi \| q_{(\alpha, \theta)}\right)=\int \log \left(\frac{\pi(x)}{\sum_{d=1}^{D} \alpha_{d} q_{d}\left(x ; \theta_{d}\right)}\right) \pi(x) \mathrm{d} x .
$$

As usual in applications of the IS methodology to Bayesian inference, the target density $\pi$ is known only up to a normalisation constant and we will focus on a self-normalised version of IS that solely requires the availability of an unnormalised version of $\pi$ (Geweke, 1989). As a side comment, note that while $\mathfrak{E}\left(\pi, q_{(\alpha, \theta)}\right)$ is a convex function of the weights $\alpha_{1}, \ldots, \alpha_{D}$ (Douc et al., 2007a), it generally fails to be so when also optimising with respect to the component parameters $\theta_{1}, \ldots, \theta_{D}$. Given that minimising (44) in $(\alpha, \theta)$ is equivalent to maximising

$$
\int \log \left(\sum_{d=1}^{D} \alpha_{d} q_{d}\left(x ; \theta_{d}\right)\right) \pi(x) \mathrm{d} x,
$$

we are facing a task that formally resembles standard mixture maximum likelihood estimation but with an integration with respect to $\pi$ replacing the empirical sum over observations.

This analogy suggests that it is possible to maximise the entropy criterion in (4) using an approach based on the principle of the EM algorithm and, in particular, the use of the augmented mixture representation (involving the indicator variables associated with each component of the mixture). 
Before providing the details of the derivation in Section 2.2, we first state below the proposed adaptive IS algorithm which we will refer to as M-PMC (for Mixture PMC) in the following. Let $\left(X_{i, t}\right)_{1 \leq i \leq N}$ and $\left(\alpha^{t, N}, \theta^{t, N}\right)$ denote, respectively, the IS sample and the estimated mixture parameters at the $t$-th iteration of the algorithm.

\section{Algorithm 1. (M-PMC Algorithm) At iteration $t$,}

1. Generate a sample $\left(X_{i, t}\right)$ from the current mixture IS proposal (3) parameterised by $\left(\alpha^{t, N}, \theta^{t, N}\right)$ and compute the normalised importance weights

$$
\bar{\omega}_{i, t}=\frac{\pi\left(X_{i, t}\right)}{\sum_{d=1}^{D} \alpha_{d}^{t, N} q_{d}\left(X_{i, t} ; \theta_{d}^{t, N}\right)} / \sum_{j=1}^{N} \frac{\pi\left(X_{j, t}\right)}{\sum_{d=1}^{D} \alpha_{d}^{t, N} q_{d}\left(X_{j, t} ; \theta_{d}^{t, N}\right)}
$$

and the mixture posterior probabilities

$$
\rho_{d}\left(X_{i, t} ; \alpha^{t, N}, \theta^{t, N}\right)=\alpha_{d}^{t, N} q_{d}\left(X_{i, t} ; \theta_{d}^{t, N}\right) / \sum_{\ell=1}^{D} \alpha_{\ell}^{t, N} q_{\ell}\left(X_{i, t} ; \theta_{\ell}^{t, N}\right),
$$

for $i=1, \ldots, N$ and $d=1, \ldots, D$.

2. Update the parameters $\alpha$ and $\theta$ as

$$
\begin{aligned}
\alpha_{d}^{t+1, N} & =\sum_{i=1}^{N} \bar{\omega}_{i, t} \rho_{d}\left(X_{i, t} ; \alpha^{t, N}, \theta^{t, N}\right) \\
\theta_{d}^{t+1, N} & =\arg \max _{\theta_{d}}\left[\sum_{i=1}^{N} \bar{\omega}_{i, t} \rho_{d}\left(X_{i, t} ; \alpha^{t, N}, \theta^{t, N}\right) \log \left\{q_{d}\left(X_{i, t} ; \theta_{d}^{t, N}\right)\right\}\right]
\end{aligned}
$$

for $d=1, \ldots, D$.

The convergence of the algorithm may be monitored by computing the so-called normalised perplexity $\exp \left(H^{t, N}\right) / N$, where $H^{t, N}=-\sum_{i=1}^{N} \bar{\omega}_{i, t} \log \bar{\omega}_{i, t}$ is the Shannon entropy of the normalised IS weights. The normalised perplexity provides an estimate of $\exp \left[-\mathfrak{E}\left(\pi, q_{\left(\alpha^{t, N}, \theta^{t, N}\right)}\right)\right]$ and, for sufficiently large $N$, it is non-decreasing with $t$.

\subsection{Detailed Derivation}

\section{Integrated Updates}

Starting from (5), assume for the moment that integration with respect to $\pi$ is feasible. In order to update the parameters of the independent IS density (3), we will take advantage of the latent variable structure that underlines the objective function (5). The resulting algorithm - still theoretical at this stage as it involves integration with respect to $\pi$-may be interpreted as an integrated EM (Expectation-Maximisation) scheme that we now describe. Let $\alpha^{t}=\left(\alpha_{1}^{t}, \ldots, \alpha_{D}^{t}\right)$ and $\theta^{t}=\left(\theta_{1}^{t}, \ldots, \theta_{D}^{t}\right)$ denote, respectively, the mixture weights and the component parameters at the $t$-th iteration of this integrated EM algorithm.

As usual in mixtures, the latent variable $Z$ is the component indicator, with values in $\{1, \ldots, D\}$ such that the joint density $f$ of $x$ and $z$ satisfies

$$
f(z)=\alpha_{z} \quad \text { and } \quad f(x \mid z)=q_{z}\left(x ; \theta_{z}\right)
$$


which produces (3) as the marginal in $x$. As in the standard EM algorithm, we can then take advantage of this latent variable representation. Since the joint density of $X$ and $Z$ is $\alpha_{z} q_{z}\left(x ; \theta_{x}\right)$, the expectation corresponding to the E step of the EM algorithm is the expected complete log-likelihood, namely, at iteration $t$ of our algorithm,

$$
\mathbb{E}_{\pi}^{X}\left[\mathbb{E}_{\left(\alpha^{t}, \theta^{t}\right)}^{Z}\left\{\log \left(\alpha_{Z} q_{Z}\left(X ; \theta_{Z}\right)\right) \mid X\right\}\right]
$$

where the inner expectation is computed under the conditional distribution of $Z$ in the mixture model given the current value $\left(\alpha^{t}, \theta^{t}\right)$ of the parameters, i.e.

$$
f(z \mid x)=\alpha_{z}^{t} q_{z}\left(x ; \theta_{z}^{t}\right) / \sum_{d=1}^{D} \alpha_{d}^{t} q_{d}\left(x ; \theta_{d}^{t}\right),
$$

while the outer expectation is under the distribution $X \sim \pi$.

The proposed updating mechanism then corresponds to setting the new parameters $\left(\alpha^{t+1}, \theta^{t+1}\right)$ equal to

$$
\left(\alpha^{t+1}, \theta^{t+1}\right)=\arg \max _{(\alpha, \theta)} \mathbb{E}_{\pi}^{X}\left[\mathbb{E}_{\left(\alpha^{t}, \theta^{t}\right)}^{Z}\left\{\log \left(\alpha_{Z} q_{Z}\left(X ; \theta_{Z}\right)\right) \mid X\right\}\right],
$$

as in the regular EM estimation of the parameters of a mixture, except for the extra expectation over $X$. It is straightforward to check that the convexity argument used for the EM algorithm also applies in this setup and, hence, that $\left(\mathfrak{E}\left(\pi, q_{\left(\alpha^{t}, \theta^{t}\right)}\right)\right)_{t \geq 1}$ is a non-decreasing sequence. Setting

$$
\rho_{d}(X ; \alpha, \theta)=\alpha_{d} q_{d}\left(X ; \theta_{d}\right) / \sum_{\ell=1}^{D} \alpha_{\ell} q_{\ell}\left(X ; \theta_{\ell}\right),
$$

the maximisation program in (9) reduces to

$$
\begin{aligned}
& \alpha^{t+1}=\arg \max _{\alpha} \mathbb{E}_{\pi}^{X}\left[\sum_{d=1}^{D} \rho_{d}\left(X ; \alpha^{t}, \theta^{t}\right) \log \left(\alpha_{d}\right)\right], \\
& \theta^{t+1}=\arg \max _{\theta} \mathbb{E}_{\pi}^{X}\left[\sum_{d=1}^{D} \rho_{d}\left(X ; \alpha^{t}, \theta^{t}\right) \log \left(q_{d}\left(X ; \theta_{d}\right)\right)\right],
\end{aligned}
$$

where the first maximisation to be carried out under the constraint that $\sum_{d=1}^{D} \alpha_{d}^{t+1}=1$. Hence,

$$
\begin{aligned}
& \alpha_{d}^{t+1}=\mathbb{E}_{\pi}^{X}\left[\rho_{d}\left(X ; \alpha^{t}, \theta^{t}\right)\right] \\
& \theta_{d}^{t+1}=\arg \max _{\theta_{d}} \mathbb{E}_{\pi}^{X}\left[\rho_{d}\left(X ; \alpha^{t}, \theta^{t}\right) \log \left(q_{d}\left(X ; \theta_{d}\right)\right)\right] .
\end{aligned}
$$

As in the regular mixture estimation problem, the resolution of this maximisation program ultimately depends on the shape of the density $q_{d}$. If $q_{d}$ belongs to an exponential family, it is easy to derive a closed-form solution for (11), which however involves expectations under $\pi$. Section 3 provides an illustration of this fact in the Gaussian case, while the non-exponential Student's $t$ case is considered in Section 4 .

\section{Approximate Updates}

To make the previous algorithm practical, adaptivity must be achieved by updating the parameters based on the previously simulated IS sample. We thus start the algorithm by arbitrarily fixing the mixture parameters $\left(\alpha^{1}, \theta^{1}\right)$ and we then sample from the resulting proposal $\sum \alpha_{d}^{1} q_{d}\left(x ; \theta_{d}^{1}\right)$ to obtain 
our initial sample $\left(X_{i, 1}\right)_{1 \leq i \leq N}$, associated with the latent variables $\left(Z_{i, 1}\right)_{1 \leq i \leq N}$ that indicate from which component of the mixture the corresponding $\left(X_{i, 1}\right)_{1 \leq i \leq N}$ have been generated. From this stage, we proceed recursively. Starting at iteration $t$ from a sample $\left(X_{i, t}\right)_{1 \leq i \leq N}$, associated with the latent variables $\left(Z_{i, t}\right)_{1 \leq i \leq N}$ and the normalised IS weights $\left(\bar{\omega}_{i, t}\right)_{1 \leq i \leq N}$ defined in (6), we denote by $\left(\alpha^{t+1, N}, \theta^{t+1, N}\right)$ the updated value of the mixture parameters.

To approximate (10) and (11), Douc et al. (2007a) proposed the following update rule:

$$
\begin{aligned}
\alpha_{d}^{t+1, N} & =\sum_{i=1}^{N} \bar{\omega}_{i, t} \mathbb{1}\left\{Z_{i, t}=d\right\} \\
\theta_{d}^{t+1, N} & =\arg \max _{\theta_{d}}\left[\sum_{i=1}^{N} \bar{\omega}_{i, t} \mathbb{1}\left\{Z_{i, t}=d\right\} \log \left\{q_{d}\left(X_{i, t} ; \theta_{d}^{t, N}\right)\right\}\right] .
\end{aligned}
$$

The computational cost of this update is of order $N$ whatever the number $D$ of components is, since the weight and the parameter of each component are updated based only on the points that were actually generated from this component. However, this observation also suggests that (12) may be highly variable when $N$ is small and/or $D$ becomes larger. To make the update more robust, we here propose a simple Rao-Blackwellisation step that consists in replacing $\mathbb{1}\left\{Z_{i, t}=d\right\}$ with its conditional expectation given $X_{i, t}$, that is, $\rho_{d}\left(X_{i, t} ; \alpha^{t, N}, \theta^{t, N}\right)$ defined in (17). The resulting parameters update is given by (8), which we selected for Algorithm 11.

Examining (77) indicates that the evaluation of the posterior probabilities $\rho_{d}\left(X_{i, t} ; \alpha^{t, N}, \theta^{t, N}\right)$ does not represent a significant additional computation cost, given that the denominator of this expression has already been computed when evaluating the IS weights according to (6). The most significant difference between (8) and (12) is that, with the former, all points contribute to the updating of the $d$-th component, for an overall cost proportional to $D \times N$. Note however that in many applications of interest, the most significant computational cost is associated with the evaluation of $\pi$ - which is performed exactly $N$ times per iteration - so that the cost of the update is mostly negligible, even with the Rao-Blackwellised version.

\section{Convergence of the M-PMC Algorithm}

Convergence of the estimated parameters as $N$ increases can be established using the same approach as in Douc et al. (2007a, $\mathrm{b})$, relying mainly on the convergence property of triangular arrays of random variables (see Theorem A.1 in Douc et al., 2007a). For the Rao-Blackwellised version, assuming that for all $\theta$ 's, $\pi\left(q_{d}\left(\cdot ; \theta_{d}\right)=0\right)=0$, for all $\alpha$ 's and $\theta$ 's, $\rho_{d}(\cdot ; \alpha, \theta) \log q_{d}\left(\cdot, \theta_{d}\right) \in L^{1}(\pi)$, and some (uniform in $x$ ) regularity conditions on $q_{d}(x ; \theta)$ viewed as a function of $\theta$, yield

$$
\alpha_{d}^{t+1, N} \stackrel{\mathbb{P}}{\rightarrow} \alpha_{d}^{t+1}, \quad \quad \theta_{d}^{t+1, N} \stackrel{\mathbb{P}}{\rightarrow} \theta_{d}^{t+1}
$$

when $N$ goes to infinity. Note that we do not expand on the regularity conditions imposed on $q_{d}$ since, for the algorithm to be efficient, we definitely need a closed-form expression on the parameter updates. It is then easier to deal with the convergence of the approximation of these update formulas on a case-by-case basis, as will be seen in the Gaussian example of Section 3 .

As a practical criterion for monitoring the convergence of the algorithm we recommend computing the normalised perplexity $\exp \left(H^{t, N}\right) / N$ (see Algorithm 1) and to interrupt adaptation when it stabilises and/or becomes sufficiently close to 1 . Note that in referring to $\exp \left(H^{t, N}\right)$ (exponential of the Shannon entropy expressed in nat) as the perplexity, we follow the terminology in use in the field of natural language processing. The connection between the perplexity and the entropy criterion (2) 
is revealed by writing

$$
\exp \left[-\mathfrak{E}\left(\pi, q_{(\alpha, \theta)}\right)\right]=\exp \left(\int-\log \frac{\pi_{\text {unn }}(x)}{q_{(\alpha, \theta)}(x)} \pi(x) \mathrm{d} x\right)\left(\int \pi_{\text {unn }}(x) \mathrm{d} x\right),
$$

where $\pi_{\text {unn }}$ refers to the unnormalised version of $\pi$ which is effectively computable. Estimating the first integral in (13) by self-normalised IS as

$$
-\sum_{i=1}^{N} \bar{\omega}_{i, t} \log \frac{\pi_{\mathrm{unn}}\left(X_{i, t}\right)}{q_{\left(\alpha^{t, N}, \theta^{t, N}\right)}\left(X_{i, t}\right)}
$$

and the second one by classical IS, as

$$
1 / N \sum_{i=1}^{N} \pi_{\mathrm{unn}}\left(X_{i, t}\right) / q_{\left(\alpha^{t, N}, \theta^{t, N}\right)}\left(X_{i, t}\right),
$$

indeed shows that $\exp \left(H^{t, N}\right) / N$ is a consistent estimator of $\exp \left[-\mathfrak{E}\left(\pi, q_{\left(\alpha^{t, N}, \theta^{t, N}\right)}\right)\right]$. The entropy of the IS weights is frequently used as a criterion for assessing the quality of an IS sample - together with the so-called Effective Sample Size (ESS) (Chen and Liu, 1996, Doucet et al., 2001, Cappé et al., 2005). To the best of our knowledge, however the strong connection between this criterion and the performance measure $\mathfrak{E}\left(\pi, q_{\left(\alpha^{t, N}, \theta^{t, N}\right)}\right)$ used in the present work had not been noted before.

\section{Variance Estimation}

For the sake of completeness, we recall here the formula by which it is possible to estimate, from the IS sample, the asymptotic variance of the IS estimate. If one considers a test function $h$ of interest, the self-normalised IS estimation of its expectation under $\pi$ is $\widehat{\pi(h)}=\sum_{i=1}^{N} \bar{\omega}_{i} h\left(X_{i}\right)$ and its asymptotic variance is given by

$$
v(h)=\int\{h(x)-\pi(h)\}^{2} \pi^{2}(x) / q_{\alpha, \theta}(x) \mathrm{d} x,
$$

under the assumption that $\int\left(1+h^{2}(x)\right) \pi^{2}(x) / q_{\alpha, \theta}(x) \mathrm{d} x<\infty$. The asymptotic variance $v(h)$ may thus be consistently estimated by $N \sum_{i=1}^{N} \bar{\omega}_{i}^{2}\left\{h\left(X_{i}\right)-\widehat{\pi(h)}\right\}^{2}$ (Geweke, 1989).

\section{The Gaussian mixture case}

As a first example, we consider the case of $p$-dimensional Gaussian mixture IS densities of the form

$$
q_{d}\left(X ; \theta_{d}\right)=\left\{(2 \pi)^{p}\left|\Sigma_{d}\right|\right\}^{-1 / 2} \exp \left\{-\frac{1}{2}\left(X-\mu_{d}\right)^{\mathrm{T}} \Sigma_{d}^{-1}\left(X-\mu_{d}\right)\right\}
$$

where $\theta_{d}=\left(\mu_{d}, \Sigma_{d}\right)$ denotes the parameters of the $d$-th Gaussian component density. This parametrisation of the IS density provides a general framework for approximating multivariate targets $\pi$ and the corresponding algorithm is a straightforward instance of the general framework discussed in the previous section.

\section{$3.1 \quad$ Update formulas}

The integrated update formulas are obtained as the solution of

$$
\theta_{d}^{t+1, N}=\arg \min _{\theta} \mathbb{E}_{\pi}^{X}\left[\rho_{d}\left(X ; \alpha^{t}, \theta^{t}\right)\left(\log \left|\Sigma_{d}\right|+\left(X-\mu_{d}\right)^{\mathrm{T}} \Sigma_{d}^{-1}\left(X-\mu_{d}\right)\right)\right] .
$$


It is straightforward to check that the infimum is reached when, for $d \in\{1, \ldots, D\}$,

$$
\mu_{d}^{t+1}=\frac{\mathbb{E}_{\pi}^{X}\left[\rho_{d}\left(X ; \alpha^{t}, \theta^{t}\right) X\right]}{\mathbb{E}_{\pi}^{X}\left[\rho_{d}\left(X ; \alpha^{t}, \theta^{t}\right)\right]},
$$

and

$$
\Sigma_{d}^{t+1}=\frac{\mathbb{E}_{\pi}^{X}\left[\rho_{d}\left(X ; \alpha^{t}, \theta^{t}\right)\left(X-\mu_{d}^{t+1}\right)\left(X-\mu_{d}^{t+1}\right)^{\mathrm{T}}\right]}{\mathbb{E}_{\pi}^{X}\left[\rho_{d}\left(X ; \alpha^{t}, \theta^{t}\right)\right]} .
$$

At iteration $t$ of the M-PMC algorithm, both the numerator and the denominator of each of the above expressions are approximated using self-normalised importance sampling. Denoting $\mathbb{1}\left\{Z_{i, t}=d\right\}$ by $\xi_{i, t}$, the following empirical update equations are obtained for the basic updating strategy (12):

$$
\begin{aligned}
\alpha_{d}^{t+1, N} & =\sum_{i=1}^{N} \bar{\omega}_{i, t} \xi_{i, t}, \\
\mu_{d}^{t+1, N} & =\frac{\sum_{i=1}^{N} \bar{\omega}_{i, t} \xi_{i, t} X_{i, t}}{\sum_{i=1}^{N} \bar{\omega}_{i, t} \xi_{i, t}}=\sum_{i=1}^{N} \bar{\omega}_{i, t} \xi_{i, t} X_{i, t} / \alpha_{d}^{t+1, N}, \\
\Sigma_{d}^{t+1, N} & =\sum_{i=1}^{N} \bar{\omega}_{i, t} \xi_{i, t}\left(X_{i, t}-\mu_{d}^{t+1, N}\right)\left(X_{i, t}-\mu_{d}^{t+1, N}\right)^{\mathrm{T}} / \alpha_{d}^{t+1, N} .
\end{aligned}
$$

For the Rao-Blackwellised scheme of Algorithm 1, the update is formally identical to the one above upon replacing $\xi_{i, t}$ by its conditional expectation

$$
\xi_{i, t}^{R B}=\rho_{d}\left(X_{i, t} ; \alpha^{t, N}, \theta^{t, N}\right) .
$$

Note that, as discussed in Section 2.2, establishing the convergence of the parameter update in this Gaussian setting will only require the assumption that $\rho_{d}(x ; \alpha, \theta) x^{2}$ is integrable with respect to $\pi$ (see Douc et al., 2007a).

\section{$3.2 \quad$ A simulation experiment}

To illustrate the results of the algorithm presented above, we consider a toy example in which the target density consists of a mixture of two multivariate Gaussian densities. The appeal of this example is that it is sufficiently simple to allow for an explicit characterisation of the attractive points for the adaptive procedure, while still illustrating the variety of situations found in more realistic applications. In particular, the model contains an attractive point that does not correspond to the global minimum of the entropy criterion as well as some regions of attraction that can eventually lead to a failure of the algorithm. The results obtained on this example also illustrate the improvement brought by the Rao-Blackwellised update formulas in (15).

The target $\pi$ is a mixture of two $p$-dimensional Gaussian densities such that

$$
\pi(x)=0.5 \mathcal{N}\left(x ;-s \mathbf{u}_{p}, \mathbf{I}_{p}\right)+0.5 \mathcal{N}\left(x ; s \mathbf{u}_{p}, \mathbf{I}_{p}\right),
$$

when $\mathbf{u}_{p}$ is the $p$-dimensional vector whose coordinates are equal to 1 and $\mathbf{I}_{p}$ stands for the identity matrix. In the sequel, we focus on the case where $p=10$ and $s=2$. Note that one should not be misled by the image given by the marginal densities of $\pi$ : in the ten dimensional space, the two components of $\pi$ are indeed very far from one another. It is for instance straightforward to check that the Kullback-Leibler divergence between the two components of $\pi, D\left\{\mathcal{N}\left(s \mathbf{u}_{p}, \mathbf{I}_{p}\right) \| \mathcal{N}\left(-s \mathbf{u}_{p}, \mathbf{I}_{p}\right)\right\}$, is equal to $\frac{1}{2}\left\|2 s \mathbf{u}_{p}\right\|^{2}=2 s^{2} p$, that is 80 in the case under consideration. In particular, were we to use 
one of the components of the mixture as an IS density for the other, we know from the arguments exposed at the end of Section 2 that the normalised perplexity of the weights would eventually tend to $\exp (-80)$. This number is so small that, for any feasible sample size, using one of the component densities of $\pi$ as an IS instrumental density for the other component or even for $\pi$ itself can only provide useless biased estimates.

The initial IS density $q_{0}$ is chosen here as the isotropic ten-dimensional Gaussian density with a covariance matrix of $5 \mathbf{I}_{p}$. The performances of $q_{0}$ as an importance sampling density, when compared to various other alternatives, are fully detailed in Table 1 below but the general comment is that it corresponds to a poor initial guess which would provide highly variable results when used with any sample size under 50,000.

\begin{tabular}{l|c|c|c} 
Proposal & N-PERP & N-ESS & $\sigma^{2}\left(x_{1}\right)$ \\
\hline$q_{0}^{\dagger}$ & $6.5 \mathrm{E}-4$ & $1.5 \mathrm{E}-4$ & $37 \mathrm{E} 3$ \\
Best fitting Gaussian $^{\dagger}$ & 0.31 & 0.27 & 19 \\
Target mixture $^{\dagger}$ & $1^{\dagger}$ & $1^{\dagger}$ & $5^{\dagger}$ \\
Best fitting Gaussian (defensive option) & 0.28 & 0.23 & 22 \\
Best fitting two Gaussian mixture (defensive option) & 0.89 & 0.87 & 5.8
\end{tabular}

Table 1: Performance of various importance sampling densities in terms of N-PERP: Normalised perplexity; N-ESS: Normalised Effective Sample Size; $\sigma^{2}\left(x_{1}\right)$ : Asymptotic variance of self-normalised IS estimator for the coordinate projection function $h(x)=x_{1}$. Quantities marked with a dagger sign are straightforward to determine, all others have been obtained using IS with a sample size of one million.

In addition to figures related to the initial IS density $q_{0}$, Table1 1 also reports performance obtained with the best fitting Gaussian IS density (with respect to the entropy criterion), which is straightforwardly obtained as the centred Gaussian density whose covariance matrix matches the one of $\pi$, that is, $\mathbf{I}_{p}+s^{2} \mathbf{u}_{p} \mathbf{u}_{p}^{\mathrm{T}}$. Of course the best possible performance achievable with a mixture of two Gaussian densities, always with the entropy criterion, is obtained when using $\pi$ as an IS density (second line of Table 1). Finally both final lines of Table 1 report the best fit obtained with IS densities of the form $0.9 \sum_{d=1}^{D} \alpha_{d} \mathcal{N}\left(\mu_{d}, \Sigma_{d}\right)+0.1 q_{0}(\cdot)$ when, respectively, $D=1$ and $D=2$ (further comments on the use of these are given below). As a general comment on Table 1, note that the variations of the perplexity of the IS weights, of the ESS and of the asymptotic variance of the IS estimate for the coordinate projection function are very correlated. This is a phenomenon that we have observed on many examples and which justifies our postulate that minimising the entropy criterion does provide very significant variance reductions for the IS estimate of "typical" functions of interest.

In this example, one may categorise the possible outcomes of adaptive IS algorithms based on mixtures of Gaussian IS densities into mostly four situations:

Disastrous (D.) After $T$ iterations of the M-PMC scheme, $q_{\left(\alpha^{T}, \theta^{T}\right)}$ is not a valid IS density (in the sense that the importance sampling unbiasedness property does not hold due to support restrictions) and may lead to inconsistent estimates. Typically, this may happen if $q_{\left(\alpha^{T}, \theta^{T}\right)}$ becomes much too peaky with light tails. As discussed above, it will also practically be the case if the algorithm only succeeds in fitting $q_{\left(\alpha^{T}, \theta^{T}\right)}$ to one of both Gaussian modes of $\pi$. Another disastrous outcome is when the direct application of the adaptation rules described above leads to numerical problems, usually due to the poor conditioning of some of the covariance matrices $\Sigma_{d}$. Rather than fixing these issues by ad-hoc solutions (eg. diagonal loading), which could nonetheless be useful in practical applications, we consider below more principled ways of making the algorithm more resistant to such failures. 
Mediocre (M.) After adaptation, $q_{\left(\alpha^{T}, \theta^{T}\right)}$ is not significantly better than $q_{0}$ in terms of the performance criteria displayed in Table 1 and, in this case, the adaptation is useless.

Good (G.) After $T$ iterations, $q_{\left(\alpha^{T}, \theta^{T}\right)}$ selects the best fitting Gaussian approximation (second line of Table 1) which already provides a very substantial improvement as it results in variance reductions by about four orders of magnitude for typical functions of interest.

Excellent (E.) After $T$ iterations, $q_{\left(\alpha^{T}, \theta^{T}\right)}$ selects the best fitting mixture of two Gaussian densities, which in this somewhat artificial example corresponds to a perfect fit of $\pi$. Note, however that, the actual gain over the previous outcome is rather moderate with a reduction of variance by a factor less than four.

Of course, a very important parameter here is the IS sample size $N$ : for a given initial IS density $q_{0}$, if $N$ is too small, any method based on IS is bound to fail, conversely when $N$ gets large all reasonable algorithms are expected to reach either the G. or E. result. Note that with local adaptive rules such as the ones proposed in this paper, it is not possible to guarantee that only the E. outcome will be achieved as the best fitting Gaussian IS density is indeed a stationary point (and in fact a local minimum) of the entropy criterion. So, depending on the initialisation, there always is a non zero probability that the algorithm converges to the G. situation only.

To focus on situations where algorithmic robustness is an issue, we purposely chose to select a rather small IS sample size of $N=5,000$ points. As discussed above, direct IS estimates using $q_{0}$ as IS density would be mostly useless with such a modest sample size. We evaluated four algorithmic versions of the M-PMC algorithm. The first, Plain M-PMC, uses the parameter update formulas in (14) and $q_{0}$ is only used as an initialisation value, which is common to all $D$ components of the mixture (which also initially have equal weights). Only the means of the components are slightly perturbed to make it possible for the adaptation procedure to actually provide distinct mixture components. One drawback of the plain M-PMC approach is that we do not ensure during the course of the algorithm that the adapted mixture IS density remains appropriate for IS approximations, in particular that it provides reliable estimates of the parameter update formulas. To guarantee that the IS weights stay well behaved, we consider a version of the M-PMC algorithm in which the IS density is of the form

$$
\left(1-\alpha_{0}\right) \sum_{d=1}^{D} \alpha_{d} \mathcal{N}\left(\mu_{d}, \Sigma_{d}\right)+\alpha_{0} q_{0}
$$

with the difference that $\alpha_{0}$ is a fixed parameter which is not adapted. The aim of this version, which we call Defensive $M-P M C$ in reference to the work of Hesterberg (1995), is to guarantee that the importance function remains bounded by $\alpha_{0}^{-1} \pi(x) / q_{0}(x)$, whatever happens during the adaptation, thus guaranteeing a finite variance. Since $q_{0}$ is a poor IS density, it is preferable to keep $\alpha_{0}$ as low as possible and we used $\alpha_{0}=0.1$ in all the following simulations. As detailed in both last lines of Table 1, this modification will typically slightly limit the performances achievable by the adaptation procedure, although this drawback could probably be avoided by allowing for a decrease of $\alpha_{0}$ during the iterations of the M-PMC. The parameter update formulas for this modified mixture model are very easily deduced from (14) and are omitted here for the sake of conciseness. The third version we considered is termed Rao-Blackwellised $M$-PMC and consists in replacing the update equations (14) by their Rao-Blackwellised version (15). Finally, we consider a fourth option in which both the defensive mixture density and the Rao-Blackwellised update formulas are used.

All simulations were carried out using a sample size of $N=5,000,20$ iterations of the M-PMC algorithm and Gaussian mixtures with $D=3$ components. Note that we purposely avoided to chose $D=2$ to avoid the very artificial "perfect fit" phenomenon. This also means that for most runs of the algorithm, at least one component will disappear (by convergence of its weight to zero) or will be duplicated, with several components sharing very similar parameters. 


\begin{tabular}{l|c|c|c|c} 
& Disastrous & Mediocre & Good & Excellent \\
\hline Plain & 55 & 0 & 33 & 12 \\
\hline Defensive & 13 & 51 & 30 & 6 \\
\hline R.-B. & 18 & 1 & 70 & 11 \\
\hline Defensive + R.-B. & 5 & 11 & 76 & 8
\end{tabular}

Table 2: Number of outcomes of each category for the four algorithmic versions, as recorded from 100 independent runs.

Table 2 display the performance of the four algorithms in repeated independent adaptation runs. The most significant observation about Table 2 is the large gap in robustness between the non RaoBlackwellised versions of the algorithm, which returned disastrous or mediocre results in about $60 \%$ of the cases, a fraction that falls bellow $20 \%$ when the Rao-Blackwellised update formulas are used. Obviously the fact that the Rao-Blackwellised updates are based on all simulated values and not just on those actually simulated from a particular mixture component is a major source of robustness of the method when the sample size $N$ is small, given the misfit of the initial IS density $q_{0}$. The same remark also applies when the M-PMC algorithm is to be implemented with a large number $D$ of components. The role of the defensive mixture component is more modest although it does improve the performance of both versions of the algorithm (non Rao-Blackwellised and Rao-Blackwellised altogether), at the price of a slight reduction of the frequency of the "Excellent" outcome. Also notice that the results obtained when the defensive mixture component is used are slightly beyond those of the unconstrained adaptation (see Table 1). The frequency of the perfect or "Excellent" match is about $10 \%$ for all methods but this is a consequence of the local nature of the adaptation rule as well as of the choice of the initialisation of the algorithm. It should be stressed however that as we are not interested in modelling $\pi$ by a mixture but rather that we are seeking good IS densities, the solutions obtained in the G. or E. situations are only mildly different in this respect (see Table 1). As a final comment, recall that the results presented above have been obtained with a fairly small sample size of $N=5,000$. Increasing $N$ quickly reduces the failure rate of all algorithms: for $N=20,000$ for instance, the failure rate of the plain M-PMC algorithm drops to 7/100 while the Rao-Blackwellised versions achieve either the G. or E. result (and mostly the G. one, given the chosen initialisation) for all runs.

\section{Robustification via mixtures of multivariate $t$ 's}

We now consider the setting of a proposal composed of a mixture of $p$-dimensional $t$ distributions,

$$
\sum_{d=1}^{D} \alpha_{d} \mathcal{T}\left(\nu_{d}, \mu_{d}, \Sigma_{d}\right) .
$$

We here follow the recommendations of West (1992) and Oh and Berger (1993) who proposed using mixtures of $t$ distributions in importance sampling. The $t$ mixture is preferable to a normal mixture because of its heavier tails that can capture a wider range of non-Gaussian targets with a smaller number of components. This alternative setting is more challenging however and one must take advantage of the missing variable representation of the $t$ distribution itself to achieve a closed-form updating of the parameters $\left(\mu_{d}, \Sigma_{d}\right)_{d}$ approximating (11), since a true closed-form cannot be derived. 


\subsection{The latent-data framework}

Using the classical normal/chi-squared decomposition of the $t$ distribution, a joint distribution associated with the $t$ mixture proposal (16) is

$$
\begin{aligned}
f(x, y, z) & \propto \alpha_{z}\left|\Sigma_{z}\right|^{-1 / 2} \exp \left\{-\left(x-\mu_{z}\right)^{\mathrm{T}} \Sigma_{z}^{-1}\left(x-\mu_{z}\right) y / 2 \nu_{z}\right\} y^{\left(\nu_{z}+p\right) / 2-1} e^{-y / 2} \\
& \propto \alpha_{z} \varphi\left(x ; \mu_{z}, \nu_{z} \Sigma_{z} / y\right) \varsigma\left(y ; \nu_{z} / 2,1 / 2\right),
\end{aligned}
$$

where, as above, $x$ corresponds to the observable in (16),$z$ corresponds to the mixture indicator, and $y$ corresponds to the $\chi_{\nu}^{2}$ completion. The normal density is denoted by $\varphi$ and the gamma density by ऽ. Both $y$ and $z$ correspond to latent variables in that the integral of the above in $(y, z)$ returns (16).

In the associated M-PMC algorithm, we only update the expectations and the covariance structures of the $t$ distributions and not the number of degrees of freedom, given that there is no closed-form solution for the later. In that case, $\theta_{d}=\left(\mu_{d}, \Sigma_{d}\right)$ and, for each $d=1, \ldots, D$, the number of degrees of freedom $\nu_{d}$ is fixed. At iteration $t$, the integrated EM update of the parameter will involve the following "E" function

$$
Q\left\{\left(\alpha^{t}, \theta^{t}\right),(\alpha, \theta)\right\}=\mathbb{E}_{\pi}^{X}\left[\mathbb{E}_{\left(\alpha^{t}, \theta^{t}\right)}^{Y, Z}\left\{\log \left(\alpha_{Z}\right)+\log \left(\varphi\left(X ; \mu_{Z}, \nu_{Z} \Sigma_{z} / Y\right)\right) \mid X\right\}\right],
$$

since the $\chi^{2}$ part does not involve the parameter $\theta=(\mu, \Sigma)$. Given that

$$
Y, Z \mid X, \theta \sim f(y, z \mid x) \propto \alpha_{z} \varphi\left(x ; \mu_{z}, \nu_{z} \Sigma_{z} / y\right) \varsigma\left(y ; \nu_{z} / 2,1 / 2\right),
$$

we have that

$$
Y \mid X, Z=d, \theta \sim \mathcal{G} a\left[\left(\nu_{d}+p\right) / 2, \frac{1}{2}\left\{1+\left(X-\mu_{d}\right)^{\mathrm{T}} \Sigma_{d}^{-1}\left(X-\mu_{d}\right) / \nu_{d}\right\}\right]
$$

and therefore

$$
\begin{aligned}
& Q\left\{\left(\alpha^{t}, \theta^{t}\right),(\alpha, \theta)\right\}=\mathbb{E}_{\pi}^{X} {\left[\sum_{d=1}^{D} \rho_{d}\left(X ; \alpha^{t}, \theta^{t}\right) \log \left(\alpha_{d}^{\prime}\right)\right] } \\
&-\frac{1}{2} \mathbb{E}_{\pi}^{X}\left[\sum _ { d = 1 } ^ { D } \rho _ { d } ( X ; \alpha ^ { t } , \theta ^ { t } ) \left\{\log \left|\Sigma_{d}\right|+\left(X-\mu_{d}\right)^{\mathrm{T}} \Sigma_{d}{ }^{-1}\left(X-\mu_{d}\right)\right.\right. \\
&\left.\left.\times \frac{\nu_{d}+p}{\nu_{d}+\left(X-\mu_{d}^{t}\right)^{\mathrm{T}}\left(\Sigma_{d}^{t}\right)^{-1}\left(X-\mu_{d}^{t}\right)}\right\}\right],
\end{aligned}
$$

where we have used both the notation,

$$
\rho_{d}\left(X ; \alpha^{t}, \theta^{t}\right)=\mathbb{P}_{\alpha^{t}, \theta^{t}}(Z=d \mid X)=\frac{\alpha_{d}^{t} t\left(x ; \nu_{d}, \mu_{d}^{t}, \Sigma_{d}^{t}\right)}{\sum_{\ell=1}^{D} \alpha_{\ell}^{t} t\left(x ; \nu_{\ell}, \mu_{\ell}^{t}, \Sigma_{\ell}^{t}\right)},
$$

with $t(x ; \nu, \mu, \Sigma)$ denoting the $\mathcal{T}(\nu, \mu, \Sigma)$ density, and the fact that

$$
\gamma_{d}\left(X ; \theta^{t}\right)=\mathbb{E}_{\theta^{t}}^{Y}\left\{Y / \nu_{d} \mid X, Z=d\right\}=\frac{\nu_{d}+p}{\nu_{d}+\left(X-\mu_{d}^{t}\right)^{\mathrm{T}}\left(\Sigma_{d}^{t}\right)^{-1}\left(X-\mu_{d}^{t}\right)} .
$$

Therefore, the "M" step of the integrated EM update is

$$
\begin{aligned}
\alpha_{d}^{t+1} & =\mathbb{E}_{\pi}^{X}\left[\rho_{d}\left(X ; \alpha^{t}, \theta^{t}\right)\right] \\
\mu_{d}^{t+1} & =\frac{\mathbb{E}_{\pi}^{X}\left[\rho_{d}\left(X ; \alpha^{t}, \theta^{t}\right) \gamma_{d}\left(X ; \theta^{t}\right) X\right]}{\mathbb{E}_{\pi}^{X}\left[\rho_{d}\left(X ; \alpha^{t}, \theta^{t}\right) \gamma_{d}\left(X ; \theta^{t}\right)\right]} \\
\Sigma_{d}^{t+1} & =\frac{\mathbb{E}_{\pi}^{X}\left[\rho_{d}\left(X ; \alpha^{t}, \theta^{t}\right) \gamma_{d}\left(X ; \theta^{t}\right)\left(X-\mu_{d}^{t+1}\right)\left(X-\mu_{d}^{t+1}\right)^{\mathrm{T}}\right]}{\mathbb{E}_{\pi}^{X}\left[\rho_{d}\left(X ; \alpha^{t}, \theta^{t}\right)\right]} .
\end{aligned}
$$


While the first update is the generic weight modification (10), the latter formulae are (up to the integration with respect to $X$ ) essentially those found in Peel and McLachlan (2000) for a mixture of $t$ distributions.

\subsection{Parameter update}

As in Section 3.1, the empirical update equations are obtained by using self-normalised IS with weights $\bar{\omega}_{i, t}$ given by (6) for both the numerator and the denominator of each of the above expressions. The Rao-Blackwellised approximation based on (8) yields

$$
\begin{aligned}
\alpha_{d}^{t+1, N} & =\sum_{i=1}^{N} \bar{\omega}_{i, t} \rho_{d}\left(X_{i, t} ; \alpha^{t, N}, \theta^{t, N}\right), \\
\mu_{d}^{t+1, N} & =\frac{\sum_{i=1}^{N} \bar{\omega}_{i, t} \rho_{d}\left(X_{i, t} ; \alpha^{t, N}, \theta^{t, N}\right) \gamma_{d}\left(X_{i, t} ; \theta^{t, N}\right) X_{i, t}}{\sum_{i=1}^{N} \bar{\omega}_{i, t} \rho_{d}\left(X_{i, t} ; \alpha^{t, N}, \theta^{t, N}\right) \gamma_{d}\left(X_{i, t} ; \theta^{t, N}\right)}, \\
\Sigma_{d}^{t+1, N} & =\frac{\sum_{i=1}^{N} \bar{\omega}_{i, t} \rho_{d}\left(X_{i, t} ; \alpha^{t, N}, \theta^{t, N}\right) \gamma_{d}\left(X_{i, t} ; \theta^{t, N}\right)\left(X_{i, t}-\mu_{d}^{t+1, N}\right)\left(X_{i, t}-\mu_{d}^{t+1, N}\right)^{\mathrm{T}}}{\sum_{i=1}^{N} \bar{\omega}_{i, t} \rho_{d}\left(X_{i, t} ; \alpha^{t, N}, \theta^{t, N}\right)},
\end{aligned}
$$

while the standard update equations, based on (12), are obtained by replacing $\rho_{d}\left(X_{i, t} ; \alpha^{t, N}, \theta^{t, N}\right)$ by $\mathbb{1}\left\{X_{i, t}=d\right\}$ in the above equations.

\subsection{Pima Indian example}

As a realistic if artificial illustration of the performances of the $t$ mixture (16), we study the posterior distribution of the parameters of a probit model. The corresponding dataset is borrowed from the MASS library of $\mathrm{R}$ ( $\mathrm{R}$ Development Core Team, 2006). It consists in the records of 532 Pima Indian women who were tested by the U.S. National Institute of Diabetes and Digestive and Kidney Diseases for diabetes. Four quantitative covariates were recorded, along with the presence or absence of diabetes. The corresponding probit model analyses the presence of diabetes, i.e.

$$
\mathbb{P}_{\beta}(y=1 \mid \mathbf{x})=1-\mathbb{P}_{\beta}(y=0 \mid \mathbf{x})=\Phi\left(\beta_{0}+\mathbf{x}^{\mathrm{T}}\left(\beta_{1}, \beta_{2}, \beta_{3}, \beta_{4}\right)\right)
$$

with $\beta=\left(\beta_{0}, \ldots, \beta_{4}\right), \mathbf{x}$ made of four covariates, the number of pregnancies, the plasma glucose concentration, the body mass index weight in $\mathrm{kg} /(\text { height in } \mathrm{m})^{2}$, and the age, and $\Phi$ corresponds to the cumulative distribution function of the standard normal. We use the flat prior distribution $\pi(\beta \mid \mathbf{X}) \propto 1$; in that case, the 5 -dimensional target posterior distribution is such that

$$
\pi(\beta \mid \mathbf{y}, \mathbf{X}) \propto \prod_{i=1}^{532}\left[\Phi\left\{\beta_{0}+\left(\mathbf{x}^{i}\right)^{\mathrm{T}}\left(\beta_{1}, \beta_{2}, \beta_{3}, \beta_{4}\right)\right\}\right]^{y_{i}}\left[1-\Phi\left\{\beta_{0}+\left(\mathbf{x}^{i}\right)^{\mathrm{T}}\left(\beta_{1}, \beta_{2}, \beta_{3}, \beta_{4}\right)\right\}\left[^{1-y_{i}}\right.\right.
$$

where $\mathbf{x}^{i}$ is the value of the covariates for the $i$-th individuals and $y_{i}$ is the response of the $i$-th individuals.

We first present some results for $N=10,000$ sample points and $T=500$ iterations on Figures 1 3. based on a mixture with 4 components and with the degrees of freedom chosen as $\nu=(3,6,9,18)$, respectively, when using the non Rao-Blackwellised version (12). The unrealistic value of $T$ is chosen purposely to illustrate the lack of stability of the update strategy when not using the RaoBlackwellised version. Indeed, as can be seen from Figure 1, which describes the evolution of the $\mu_{d}$ 's, some components vary quite widely over iterations, but they also correspond to a rather stable overall estimate of $\beta, \sum_{i=1}^{N} \bar{\omega}_{i, T} \beta^{i, T}$, equal to $(-5.54,0.051,0.019,0.055,0.022)$ over most iterations. 

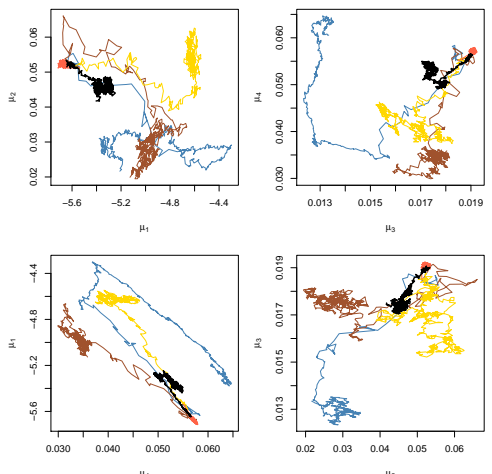

Figure 1: Pima Indians: Evolution of the components of the five $\mu_{d}$ 's over 500 iterations plotted by pairs: (clockwise from upper left side) $(1,2),(3,4),(4,1)$ and $(2,3)$. The colour code is blue for $\mu_{1}$, yellow for $\mu_{2}$, brown for $\mu_{3}$ and red for $\mu_{4}$. The additional dark path corresponds to the estimate of $\beta$. All $\mu_{d}$ 's were started in the vicinity of the MLE $\hat{\beta}$.
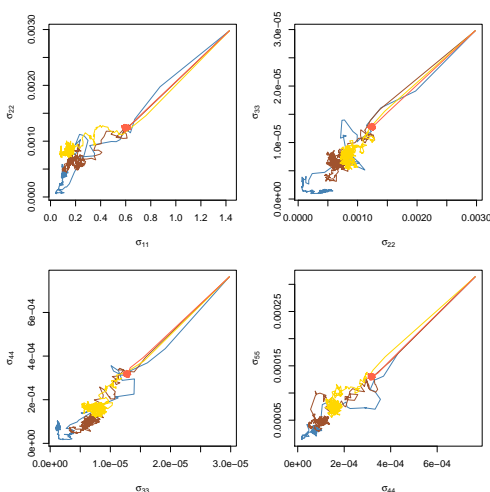

Figure 2: Pima Indians: Evolution of the five $\Sigma_{d}$ 's over 500 iterations plotted by pairs for the diagonal elements: (clockwise from upper left side) $(1,2),(3,4),(4,1)$ and $(2,3)$. The colour code is blue for $\Sigma_{1}$, yellow for $\Sigma_{2}$, brown for $\Sigma_{3}$ and red for $\Sigma_{4}$. All $\Sigma_{d}$ 's were started at the covariance matrix of $\hat{\beta}$ produced by $\mathrm{R}$ glm() procedure.
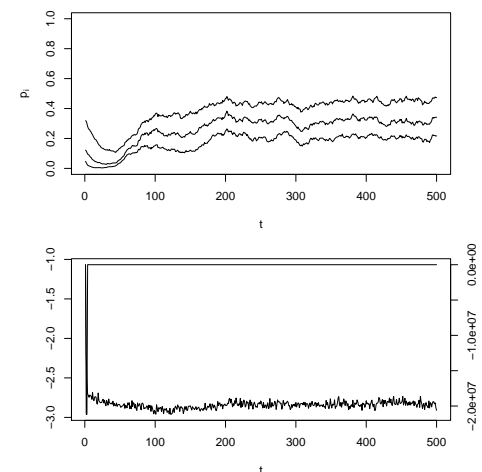

Figure 3: Pima Indians: Evolution of the cumulated weights (top) and of the estimated entropy divergence $\mathbb{E}^{\pi}\left[\log \left(q_{\alpha, \theta}(\beta)\right)\right]$ (bottom). 
When looking at Figure 3, the quasi-constant entropy estimate after iteration 100 or so shows that, even in this situation, there is little need to perpetuate the iterations till the 500-th.

Using a Rao-Blackwellised version of the updates shows a strong stabilisation for the updates of the parameters $\alpha_{d}$ and $\left(\mu_{d}, \Sigma_{d}\right)$, both in the number of iterations and in the range of the parameters. The approximation to the Bayes estimate is obviously very close to the above estimation $(-5.63,0.052,0.019,0.056,0.022)$. Figures 4 and 5 show the immediate stabilisation provided by the Rao-Blackwellisation step. In this example, which is quite typical in this respect, we recommend to use less than $T=10$ iterations in order to reserve most of the computational effort for increasing $N$, which is essential during the first adaptation steps (because the initial IS density is poor) and for the accuracy of the IS approximation in the final steps of the algorithm. Comparing the plain and Rao-Blackwellised update formulas, will really depend on how costly the parameter update is - and thus on the dimension of the model - compared to the other computational costs, and in particular the evaluation of the likelihood, which mostly depends on the number of observations. In the present case, the increase in run-time due to the use the Rao-Blackwellised formulas instead of the plain ones is negligible.

\section{Conclusions}

The M-PMC algorithm provides a flexible and robust framework for adapting general importance sampling densities represented as mixtures. The extension to mixtures of $t$ distribution broadens the scope of the method by allowing approximation of heavier tail targets. Moreover, we can extend here the remarks made in Douc et al. (2007a, b) , namely that the update mechanism provides an early stabilisation of the parameters of the mixture. It is therefore unnecessary to rely on a large value of $T$ : with large enough sample sizes $N$ at each iteration - especially on the initial iteration that requires many points to counter-weight a potentially poor initial proposal-, it is quite uncommon to fail to spot a stabilisation of both the estimates and of the entropy criterion within a few iterations.

While this paper relies on the generic entropy criterion to update the mixture density, we want to stress that it is also possible to use a more focussed deviance criterion, namely the $h$-entropy

$$
\mathfrak{E}_{h}\left(\pi, q_{(\alpha, \theta)}\right)=D\left(\pi_{h} \| q_{(\alpha, \theta)}\right)
$$

with

$$
\pi_{h}(x) \propto|h(x)-\pi(h)| \pi(x),
$$

that is tuned to the estimation of a particular function $h$, as it is well-known that the optimal choice of the importance density for the self-normalised importance sampling estimator is exactly $\pi_{h}$. Since the normalising constant in $\pi_{h}$ does not need to be known, one can derive an adaptive algorithm which resembles the method presented in this paper. It is expected that this modification will be helpful in reaching IS densities that provide a low approximation error for a specific function $h$, which is also a desirable feature of importance sampling in several applications.

\section{References}

Cappé, O., Guillin, A., Marin, J., and Robert, C. (2004). Population Monte Carlo. J. Comput. Graph. Statist., 13(4):907-929.

Cappé, O., Moulines, E., and Rydén, T. (2005). Inference in Hidden Markov Models. Springer-Verlag, New York. 

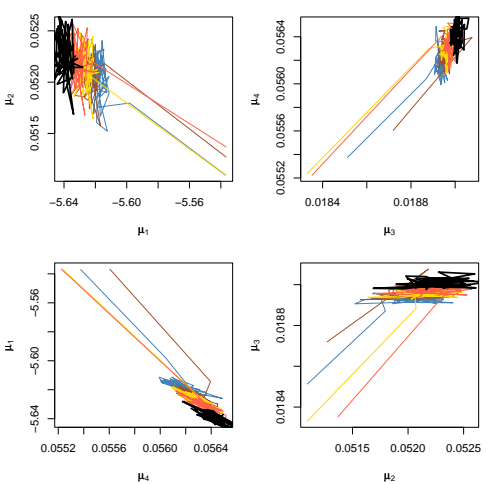

Figure 4: Pima Indians: Evolution of the components of the five $\mu_{d}$ 's over 50 Rao-Blackwellised iterations plotted by pairs: (clockwise from upper left side) $(1,2),(3,4),(4,1)$ and $(2,3)$. The colour code is blue for $\mu_{1}$, yellow for $\mu_{2}$, brown for $\mu_{3}$ and red for $\mu_{4}$. The additional dark path corresponds to the estimate of $\beta$. All $\mu_{d}$ 's were started in the vicinity of the MLE $\hat{\beta}$.
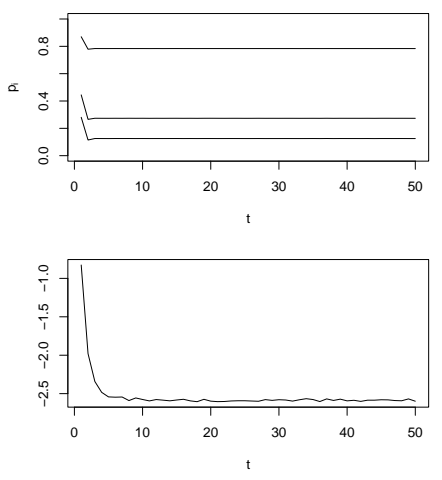

Figure 5: Pima Indians: Evolution of the cumulated weights (top) and of the estimated entropy divergence $\mathbb{E}^{\pi}\left[\log \left(q_{\alpha, \theta}(\beta)\right)\right]$ (bottom) for the Rao-Blackwellised version. 
Chen, R. and Liu, J. S. (1996). Predictive updating method and Bayesian classification. J. Royal Statist. Soc. Series B, 58(2):397-415.

Douc, R., Guillin, A., Marin, J.-M., and Robert, C. (2007a). Convergence of adaptive mixtures of importance sampling schemes. Ann. Statist., 35(1):420-448.

Douc, R., Guillin, A., Marin, J.-M., and Robert, C. (2007b). Minimum variance importance sampling via population Monte Carlo. ESAIM: Probability and Statistics, 11:427-447.

Doucet, A., de Freitas, N., and Gordon, N. (2001). Sequential Monte Carlo Methods in Practice. Springer-Verlag, New York.

Geweke, J. (1989). Bayesian inference in econometric models using Monte Carlo integration. Econometrica, 57:1317-1340.

Hesterberg, T. (1995). Weighted average importance sampling and defensive mixture distributions. Technometrics, 37(2):185-194.

Oh, M. and Berger, J. (1993). Integration of multimodal functions by Monte Carlo importance sampling. J. American Statist. Assoc., 88:450-456.

Peel, D. and McLachlan, G. (2000). Robust mixture modelling using the $t$ distribution. Statistics and Computing, 10:339-348.

R Development Core Team (2006). R: A Language and Environment for Statistical Computing. R Foundation for Statistical Computing, Vienna, Austria.

Robert, C. and Casella, G. (2004). Monte Carlo Statistical Methods. Springer-Verlag, New York, second edition.

Rubinstein, R. Y. and Kroese, D. P. (2004). The Cross-Entropy Method. Springer-Verlag, New York.

West, M. (1992). Modelling with mixtures. In Berger, J., Bernardo, J., Dawid, A., and Smith, A., editors, Bayesian Statistics 4, pages 503-525. Oxford University Press, Oxford. 